Ocean Engineering

September 2016, Volume 123 Pages 303-313

http://dx.doi.org/10.1016/.oceaneng.2016.07.026

http://archimer.ifremer.fr/doc/00347/45813/

(C) 2016 Elsevier Ltd. All rights reserved.

\title{
Finite element model for the assessment of the mesh resistance to opening of fishing nets
}

\author{
Morvan Barthelemy ${ }^{1,{ }^{*}}$, Priour Daniel ${ }^{1}$, Guede Zakoua ${ }^{2}$, Bles G. ${ }^{3}$ \\ ${ }^{1}$ French Inst Marine Res IFREMER, F-29280 Plouzane, France. \\ ${ }^{2}$ Bur Veritas, F-92200 Neuilly Sur Seine, France. \\ ${ }^{3}$ IRDL, FRE CNRS 3744, ENSTA Bretagne, F-29200 Brest, France. \\ *Corresponding author : Barthelemy Morvan, email address : barthelemy.morvan@ifremer.fr
}

\begin{abstract}
:
The evaluation of the mesh resistance to opening of fishing nets is an important issue in assessing the selectivity of trawls by numerical methods. Sala et al. (2007) proposed a method using a relatively expensive experimental device ensuring the 2D deformation of net sample. De la Prada and Gonzales (2015) proposed a simple uni-axial experimental set-up, which stretches a sample in the transverse direction of the meshes while leaving free its deformation in the normal direction. Both authors (Sala and De la Prada) assumed that the deformation is uniform in the sample. The present study aims at developing a finite element model taking into account the mesh resistance to opening of nets, allowing the simulation of non-uniform deformation. Mechanical experimental tests were performed on a range of fishing nets commonly used in trawl codends, with varied dimensions of the sample and loading. The proposed model is in good agreement with these experimental results, and it captures the heterogeneous deformation of the netting samples. Consequently, a procedure for the assessment of the mesh resistance to opening using this model and a simple non-expensive experimental setup are proposed.
\end{abstract}

\section{Highlights}

- A finite element model for the deformation of plane fishing nets was developed. The proposed model allows non-uniform deformation. Varied netting sample and sizes were tested on mechanical suspension. The finite element model was compared with test results, and El values obtained.

Keywords : Netting, Mesh resistance to opening, Bending stiffness, Experiment, Numerical model 


\section{Nomenclature}

$\begin{array}{ll}|\cdot \| \cdot| & \text { norm operator } \\ \langle\cdot, \cdot\rangle \quad\langle\cdot, \cdot\rangle & \text { scalar product } \\ \cdot \wedge \cdot \wedge \cdot & \text { cross product of vectors } \\ \delta \cdot \delta \cdot & \text { infinitesimal variation of a given variable } \\ \alpha & \text { angle between two consecutive bar elements } \\ F & \text { vector of nodal forces } \\ F_{n} & \text { nodal force at node number } n \\ \mathrm{~F}_{\mathrm{x}, \mathrm{n}} \mathrm{Fx}, \mathrm{n} & \text { nodal force at node number } n \text { in the } x \text {-direction } \\ W_{e} & \text { external work } \\ W_{i} & \text { internal work } \\ X & \text { vector of nodal positions } \\ X_{n} & \text { position of node number } n \\ X_{m n} & \text { vector connecting node number } m \text { to node number } n \\ X_{n} & \text { displacement of node number } n \text { in the } x \text {-direction }\end{array}$




\section{Introduction}

Nowadays, in some trawl fisheries codends are made from stiffer materials (Herrmann and O'Neill, 2006, Herrmann et al., 2013): double twine instead of

5 single twine, larger diameter of twine. An increase in twine bending stiffness increases the mechanical resistance of meshes to opening (Sala et al., 2007). This increased resistance affects the mesh opening in fishing gear, and thus the ability of fish to escape. Netting bending stiffness may significantly affect species selectivity (Boerema, 1956). The strong influence of the codend on the trawl selectivity has been demonstrated (Robertson and Stewart, 1988), even though selectivity in other parts of trawls is increasingly taken into account Broadhurst et al. 2015). Previous studies have shown how mesh resistance to opening affects the mechanical behaviour, and thus the selectivity of codends Herrmann and O'Neill, 2006; Sala et al. 2007). Likewise, Moderhak (2007) theoretically demonstrated how changes in mesh size and mesh resistance to opening can impact the shape of a codend and its selectivity. From a theoretical investigation, O'Neill (2003) demonstrated how an increase in twine bending stiffness reduces the diameter of the codend and thus the lateral mesh opening. Bending stiffness may be of significant effects on fish farms: during aquaculture pens towing, the netting sheets parallel and near parallel to the flow experience significant vibration, which in parts is determined by the netting bending stiffness (Johnson and Balash, 2015). Furthermore, bending stiffness is a critical factor to ensure accurate model-test drag measurements on trawl nets (Balash et al., 2016).

Considering these points, it is worthwhile to be able to measure the mesh resistance to opening of fishing nets and in particular of trawl codend nets. The mesh resistance to opening is quite difficult to evaluate. Several authors proposed to model the mesh opening by the bending of the twines. They proposed theoretical models in order to assess the bending stiffness by inverse identification. One approach is to consider that a mesh side has the same behaviour as 30 a beam (O'Neill (2002), Sala et al. (2007), De la Prada and Gonzales (2015), Priour (2013)). To evaluate the bending stiffness of mesh sides, Sala et al. 
(2007) proposed a method based on a prototype experimental device on which netting panels were mounted and deformed. The prototype incorporated four tension load cells and four stepping motors and was designed so that all the mesh sides of the netting panel would undergo the same deformation. Then, the bending stiffness was estimated from the data of forces and deformations using the beam theory. However, the prototype used a relatively expensive and complicated device to ensure the uniform deformation of the netting panel. Alternatively, Priour and Cognard (2011) assumed that the twine bending stiffness could be given by the stiffness of a simple cantilever beam with a deformation equaling the observed deformation of a netting panel subjected to out-of-plan bending. However, the method did not take into account the knot effect and required a sample of closed mesh netting (a sample with small mesh opening). De la Prada and Gonzales (2015) proposed a simple uni-axial experimental set-

45 up, which stretched a netting sample in the T-direction of the meshes (ISO, 1974) while leaving it free to deform in the N-direction. They assumed that the deformation was identical in all the meshes of the sample. However the panel was held in the vertical position during the experiment, so forces and deformations experienced by meshes at the top of the panel were higher than those supported at the bottom due to gravity.

To overcome the drawbacks of the previous methods (uniform deformation in the case of Sala et al. (2007) and De la Prada and Gonzales (2015), closed meshes in the case of Priour and Cognard (2011) $)$, we propose a finite element model for netting deformation. Using this model, uniform deformation of the netting panel or closed meshes is not required, and large deformation is allowed. The idea is, by means of this model, a simple and non-expensive experimental device can be used to evaluate the bending stiffness of mesh sides by inverse identification. The numerical model has been mainly devoted to diamond meshes because they are widely used in trawl fisheries (Herrmann et al. 2015). However, it could be 60 applied for any kind of meshes (hexagonal, square) having mesh sides presenting bending stiffness.

We performed experimental mechanical tests on a range of fishing nets com- 
monly used in trawl codends, with varied dimensions of the sample and loading.

This experimental test is the same as presented by De la Prada and Gonzales

65 (2015). The numerical simulations of these tests, by means of the proposed model, are compared with these experimental results. These numerical simulations resulted in inverse identification of the bending stiffness of the various tested fishing nets at different levels of loading.

The paper is organised as follows. Firstly, the finite element model is de70 scribed. Secondly, its validity is investigated by comparison with the experimental results. Finally, the identified bending stiffnesses are presented. 


\section{Numerical model}

A netting structure is discretized into bar elements, the extremities of which are denoted nodes and are subject to forces. When the geometry of the netting structure, the external forces acting on it and the stiffness of the mesh sides and knots are known, the equilibrium position is obtained with the following steps:

- Step 1. First, an initial position of the structure (i.e. an initial position of the nodes) is determined.

- Step 2. Then, the forces on those nodes are calculated depending on the nodal positions.

- Step 3. Finally, the equilibrium position of the structure is obtained using an incremental iterative method such as the Newton-Raphson scheme (Ortega and Rheinboldt, 2000).

The numerical model proposes specific modelling, respectively for the aligned elements and for the non-aligned elements of the structure at rest.

A mesh side connects two knots and is modelled as a beam. And more precisely, with this original model, a mesh side is modelled as an assembly of aligned elements (Section 2.1). In this document, the mesh is generally assumed to be diamond, and the knot is represented as a node and can be modelled with only non-aligned elements (Section 2.2). This model can also be used for square meshes. In this case the square is considered as a diamond with a hanging ratio of 0.707 and the same modelling is used: an assembly of aligned elements for the mesh sides and non-aligned elements for the knots. When the knots are large-sized, it could be worth considering the mesh as hexagonal. In this case aligned and non-aligned elements are used to model the knots.

\subsection{Aligned elements to model mesh sides}

The numerical model of a mesh side is based on Priour (2013). In this model, a mesh side is assumed to behave like a beam and is discretized into bar elements (Fig. 1 and Fig. 2). These elements are aligned and of equal length at rest. 


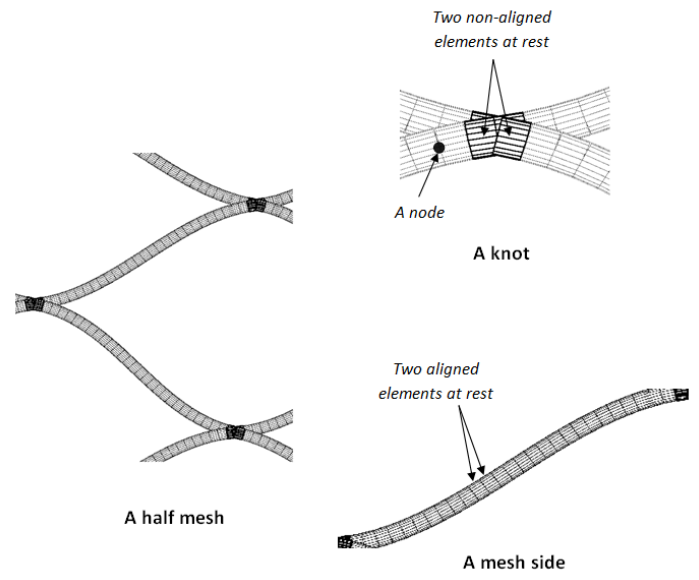

Figure 1: Netting model. A knot is modelled with, at least, two non-aligned elements at rest (diamond meshes), and mesh sides are modelled with aligned elements.

100

$$
\text { consecte }
$$

\section{conse}

the basic equation giving the curvature of an elastic beam subject to a specific moment:

$$
C=\frac{E I}{R}
$$

where $E$ is the Young's modulus, I is the moment of inertia and $\mathrm{R}$ is the radius of curvature of the beam centreline at the node under consideration. The product $E I$ stands for the bending stiffness of the beam.

However, Equation (1) is valid under the strong assumptions of a symmetrical beam, the transverse sections of which remain plane and normal to the 110 centreline after bending. Even if these assumptions are not generally fulfilled in the case of a netting, Eq. 11] is still used for the sake of simplicity.

The radius of curvature $R$ is given by the radius of the circumcircle of the triangle defined by two consecutive bar elements (Fig. 3). The radius $R$ of the 

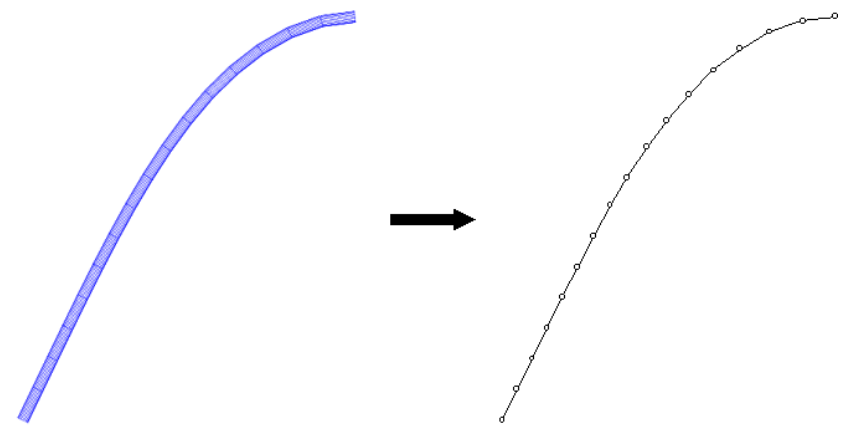

Figure 2: Mesh side discretized into bar elements. A bar element connects two nodes.

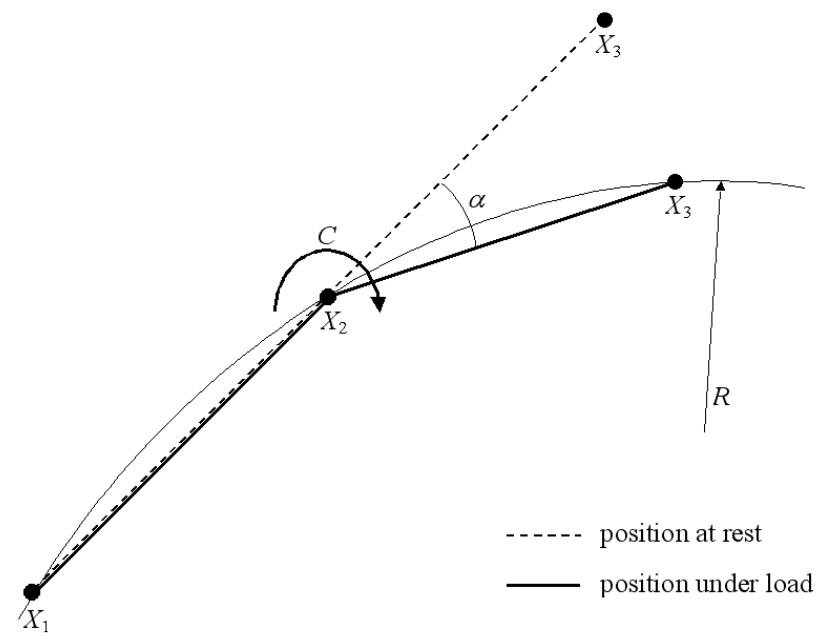

Figure 3: Two consecutive bar elements in a mesh side. When the angle $\alpha$ between elements increases, the couple $C$ increases.

circumcircle of triangle 123 is given by the formula :

$$
R=\frac{\left|X_{12}\right|\left|X_{23}\right|\left|X_{31}\right|}{4 \sqrt{p\left(p-\left|X_{12}\right|\right)\left(p-\left|X_{23}\right|\right)\left(p-\left|X_{31}\right|\right)}}
$$

where $p$ is the semiperimeter of the circumcircle:

$$
p=\frac{\left|X_{12}\right|+\left|X_{23}\right|+\left|X_{31}\right|}{2}
$$

Once the couple $C$ is calculated, the forces on the nodes are expressed using 


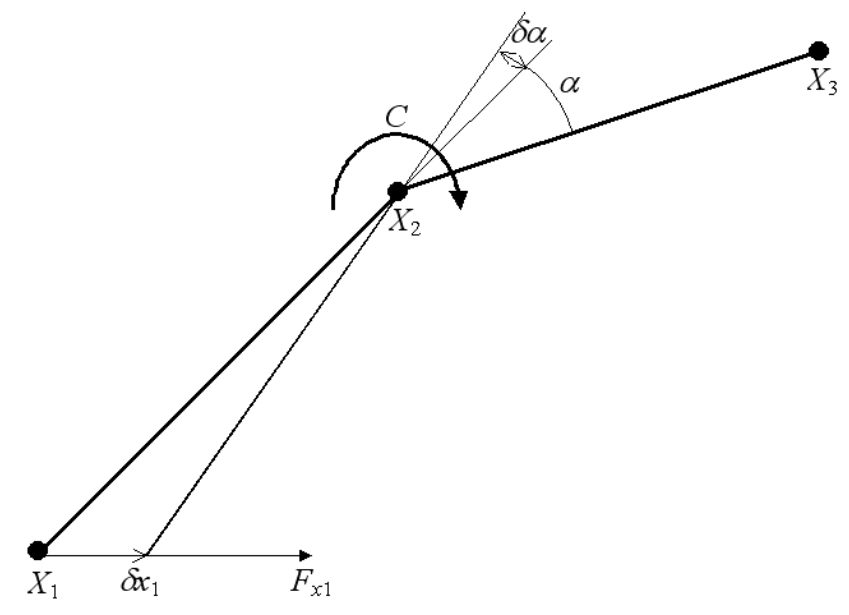

Figure 4: Virtual work principle. $\delta x_{1}$ generates an external work $F_{x, 1} \delta x_{1}$ and an internal work $C \delta \alpha$.

the virtual work principle. Let us consider a virtual displacement $\delta x_{1}$ of the node 1 along the $x$-axis (Fig. 4). That displacement produces an external virtual work $\delta W_{e}=F_{x, 1} \delta x_{1}$. It causes a variation $\delta \alpha$ of the angle $\alpha$ between the two consecutive bar elements and generates an internal virtual work $\delta W_{i}=C \delta \alpha$. ${ }_{120}$ From the basic virtual work equation, which is $\delta W_{e}=\delta W_{i}$, one obtains:

$$
F_{x, 1}=C \frac{\delta \alpha}{\delta x_{1}}=C \frac{\partial \alpha}{\partial x_{1}}
$$

Doing the same for all the nodes 1,2 and 3 and along the $x, y$ and $z$-axis, one obtains the equations:

$$
\begin{aligned}
F_{x, 1} & =C \frac{\partial \alpha}{\partial x_{1}} ; F_{x, 2}=C \frac{\partial \alpha}{\partial x_{2}} ; F_{x, 3}=C \frac{\partial \alpha}{\partial x_{3}} \\
F_{y, 1} & =C \frac{\partial \alpha}{\partial y_{1}} ; F_{y, 2}=C \frac{\partial \alpha}{\partial y_{2}} ; F_{y, 3}=C \frac{\partial \alpha}{\partial y_{3}} \\
F_{z, 1} & =C \frac{\partial \alpha}{\partial z_{1}} ; F_{z, 2}=C \frac{\partial \alpha}{\partial z_{2}} ; F_{z, 3}=C \frac{\partial \alpha}{\partial z_{3}}
\end{aligned}
$$

The partial derivatives of the angle $\alpha$ in terms of the nodal coordinates are 
obtained from the identity:

$$
\cos \alpha=\frac{<X_{12}, X_{23}>}{\left|X_{12}\right|\left|X_{23}\right|}
$$

$$
\begin{aligned}
& F_{1}=\frac{E I}{\left|X_{12}\right|\left|X_{23}\right| R \sin \alpha}\left[\frac{<X_{12}, X_{23}>X_{12}}{\left|X_{12}\right|^{2}}-X_{23}\right] \\
& F_{2}=\frac{E I}{\left|X_{12}\right|\left|X_{23}\right| R \sin \alpha}\left[-\frac{<X_{12}, X_{23}>X_{12}}{\left|X_{12}\right|^{2}}+\frac{<X_{12}, X_{23}>X_{23}}{\left|X_{23}\right|^{2}}+X_{23}-X_{12}\right]
\end{aligned}
$$

$F_{3}=\frac{E I}{\left|X_{12}\right|\left|X_{23}\right| R \sin \alpha}\left[-\frac{<X_{12}, X_{23}>X_{23}}{\left|X_{23}\right|^{2}}+X_{12}\right]$

\subsection{Non-aligned elements to model knots}

Let us now consider a node corresponding to a knot of the netting (node 2 in Fig. 5). From the netting structure, one can see that the angle at rest between the two consecutive elements on either side of this node is non-zero (Fig. 1 and

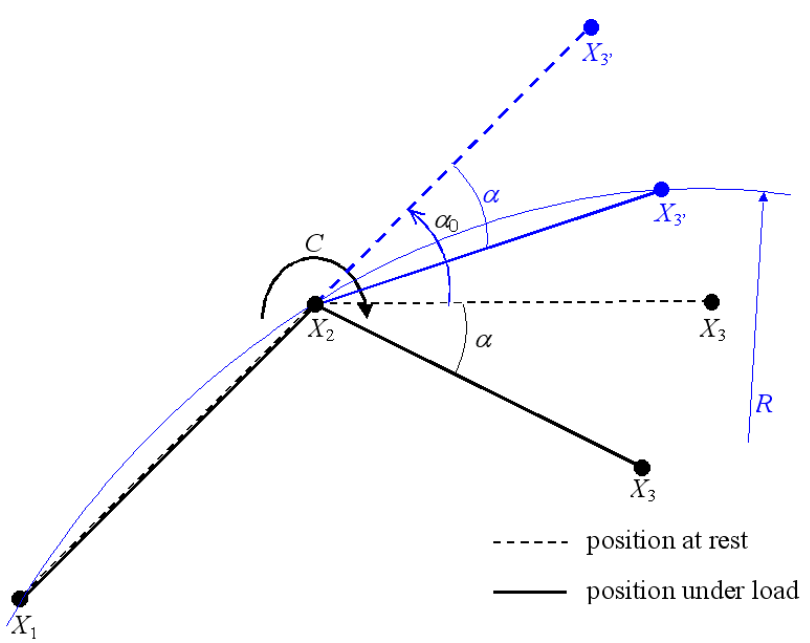

Figure 5: The couple acting on the node 2. The angle at rest is not null $\left(\alpha_{0}\right)$. 
To express the couple on such a node (node 2 in Fig. 5), one builds a virtual bar element 23'. To obtain the virtual node 3', node 3 is rotated by the angle $\alpha_{0}$ around node 2 , where $\alpha_{0}$ is the angle at rest between the two bar elements (Fig. 5):

$$
X_{3^{\prime}}=X_{2}+\mathbf{R}\left(X_{3}-X_{2}\right)
$$

where $\mathbf{R}$ is the rotation matrix by the angle $\alpha_{0}$ around the axis normal to the plan $\left(X_{12}, X_{23}\right)$ around the node $X_{2}$ and it reads:

$$
\mathbf{R}=\left(\begin{array}{ccc}
n_{x}^{2}+\left(1-n_{x}^{2}\right) c & n_{x} n_{y}(1-c)+n_{z} s & n_{x} n_{z}(1-c)-n_{y} s \\
n_{x} n_{y}(1-c)+n_{z} s & n_{y}^{2}+\left(1-n_{y}^{2}\right) c & n_{y} n_{z}(1-c)+n_{x} s \\
n_{x} n_{z}(1-c)-n_{y} s & n_{y} n_{z}(1-c)+n_{x} s & n_{z}^{2}+\left(1-n_{z}^{2}\right) c
\end{array}\right)
$$

where,

$$
\begin{gathered}
c=\cos \alpha_{0}, \\
s=\sin \alpha_{0}, \\
\left(\begin{array}{c}
n_{x} \\
n_{y} \\
n_{z}
\end{array}\right)=\frac{X_{21} \wedge X_{23}}{\left|X_{21} \wedge X_{23}\right|}
\end{gathered}
$$

Moreover, the couple at node 2 is assumed to be completely carried by the bar elements 12 and 23' while node 3 is rigidly connected to node 3'. Therefore, the couple at node 2 can be expressed by Equation (1) on the bar elements 12 and 23'. One only has to replace point $X_{3}$ by point $X_{3^{\prime}}$ in Equation 2 of the curvature radius.

Then, the expression of nodal forces in this case is obtained by replacing the curvature radius in Equation (7) : 


$$
\begin{aligned}
& F_{1}=\frac{2 E I}{\left|X_{12}\right|\left|X_{23^{\prime}}\right| R \sin \alpha}\left[\frac{<X_{12}, X_{23}>X_{12}}{\left|X_{12}\right|^{2}}-X_{23}\right] \\
& F_{2}=\frac{2 E I}{\left|X_{12}\right|\left|X_{23^{\prime}}\right| R \sin \alpha}\left[-\frac{<X_{12}, X_{23}>X_{12}}{\left|X_{12}\right|^{2}}+\frac{<X_{12}, X_{23}>X_{23}}{\left|X_{23}\right|^{2}}+X_{23}-X_{12}\right]
\end{aligned}
$$

$$
F_{3}=\frac{2 E I}{\left|X_{12}\right|\left|X_{23^{\prime}}\right| R \sin \alpha}\left[-\frac{<X_{12}, X_{23}>X_{23}}{\left|X_{23}\right|^{2}}+X_{12}\right]
$$

The vector $X_{23^{\prime}}$ is easily calculated using Equation (8).

\subsection{Equilibrium position of the netting}

Once the equations of all the nodal forces in terms of nodal positions are formulated, one can solve them using an iterative procedure such as the NewtonRaphson scheme for instance. Thus, from a defined initial position $X^{0}$, position $X$ is iteratively adapted until equilibrium is reached. In fact, one can only find an approximate solution by this method, therefore the equilibrium position is considered to have been reached when the total nodal force $F(X)$ is close enough to zero within an acceptable tolerance.

The iterative incrementation of $X$ is given by the following recurrence relation formula:

$$
\begin{aligned}
X^{(0)} & =X^{0} \\
X^{(k+1)} & =X^{(k)}+\Delta X^{(k)}
\end{aligned}
$$

The iterative increment reads:

$$
\begin{aligned}
& \Delta X^{(k)}=-\left[\frac{\partial F}{\partial X}\left(X^{(k)}\right)\right]^{-1} F\left(X^{(k)}\right) \\
& {\left[\frac{\partial F}{\partial X}\right] \text { is the tangent stiffness matrix }}
\end{aligned}
$$

\subsection{Applicability to hexagonal meshes}

Modelling diamond meshes is possible using the models of aligned elements and non-aligned elements previously described. Modelling hexagonal meshes is 
also possible. In the case of diamond meshes, 2, 3 or 4 non-aligned elements connected to one node are used to model a knot (Fig. 11). To model the knots of hexagonal meshes, non-aligned and possibly aligned elements are used. In fact, in this case, a knot can be modelled as a beam (aligned elements at rest) connected to 4 mesh sides with non-aligned elements. In Fig. 6, non-aligned elements are used 3 times to connect the half knots to the two mesh sides: between the first mesh side and the knot, between the second mesh side and the knot and between the two mesh sides.

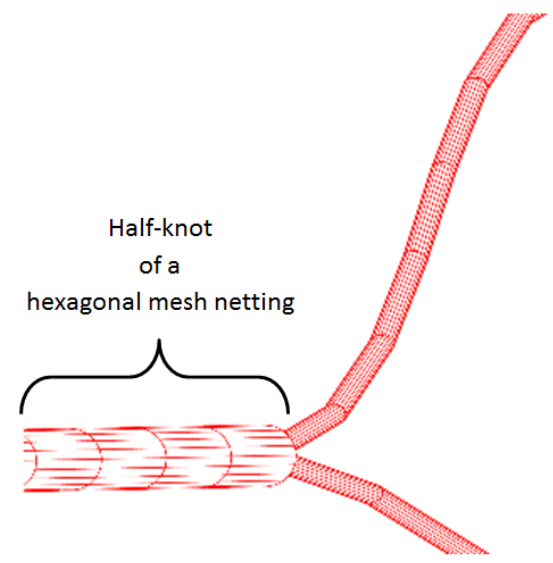

Figure 6: A half knot and two mesh sides in the case of hexagonal meshes. The model of non-aligned elements is used up to 3 times to connect a knot to 2 mesh sides: between the first mesh side and the knot, between the second mesh side and the knot and finally between the two mesh sides.

In Fig. 7. there is a modelling of netting made up of 8 by 10 hexagonal meshes which is not equilibrated at the top and equilibrated at the bottom. The netting sample is loaded by gravitational forces only, and the top boundary is fixed vertically and free horizontally.

The hexagonal sides made up of twines are $0.05 \mathrm{~m}$ long, a diameter of 0.004 m, a density of $1100 \mathrm{Kg} \cdot \mathrm{m}^{-3}$, and a bending stiffness EI of $10^{-5} \mathrm{~N} \cdot \mathrm{m}^{2}$ which is an acceptable value for twines made of polyethylene. They are modelled with 
7 bar elements. The horizontal sides that represent the knots are $0.025 \mathrm{~m}$ long, a diameter of $0.009 \mathrm{~m}$, a density of $1100 \mathrm{Kg} \cdot \mathrm{m}^{-3}$, and a bending stiffness EI of $10^{-4} N . m^{2}$. They are modelled with 5 bar elements.
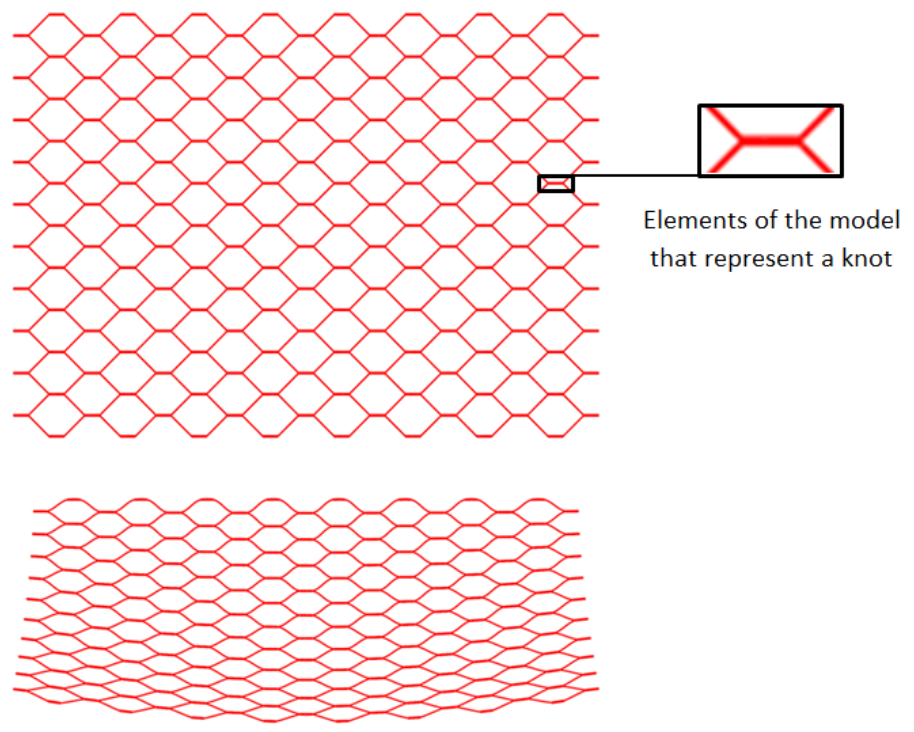

Figure 7: Netting made of hexagonal meshes, not equilibrated at the top and equilibrated at the bottom. The top boundary is fixed vertically and can slide horizontally. The netting sample is loaded by its own weight.

\section{Netting samples and experimental method}

\subsection{Netting samples for experiments}

The tested netting samples were produced by Le Drezen company (F-29730 Le Guilvinec, France). The netting types were those commonly used in trawl codends by pelagic or demersal fisheries. The netting samples were made of two materials: polyethylene (PE) or polyamide (PA); two kinds of mesh sides were used: single twine or double twine; two sizes of panel were used: 4x10-mesh or $5 \times 25$-mesh panels.

Six types of samples were used: 
- Single twine green PE netting, 4x10-mesh sample (Section 3.1.1)

- Double twine green PE netting, 4x10-mesh sample (Section 3.1.1)

- Double twine green PE netting, 5x25-mesh sample (Section 3.1.1)

- Single twine Breztop PE netting, 4x10-mesh sample (Section 3.1.2

- Single twine Brezline PE netting, 4x10-mesh sample (Section 3.1.3)

- Single twine PA netting, 4x10-mesh sample (Section 3.1.4

In order to evaluate the input data of the numerical model, the following sample characteristics were measured:

- The mass $m$ of the netting panel.

- The initial width $H_{N 0}$ and height $H_{T 0}$ of the netting panel (i.e. at rest) in the $\mathrm{N}$-direction and the $\mathrm{T}$-direction respectively. The panel position at rest was obtained by laying the netting panel free of load on a vibrating horizontal plane, where it remained free of load, until a stable position was reached.

- The characteristic length of the netting mesh sides and the mesh angle $\alpha_{0}$ (angle between two mesh sides) at rest were derived from a simple cosinus equation and the initial dimensions of the panel (Fig. 8).

\subsubsection{Green polyethylene braided netting}

We investigated single and double twine netting made of green polyethylene.

The mesh sides of the single twine netting were composed of only one polyethylene-fibre braided twine. While the mesh sides of the double twine netting were composed of two polyethylene-fibre braided twines.

The green polyethylene-fibre braided twine is the basic polyethylene product of Le Drezen company.

The diameter of the twine was $3.14 \pm 0.1 \mathrm{~mm}$. 


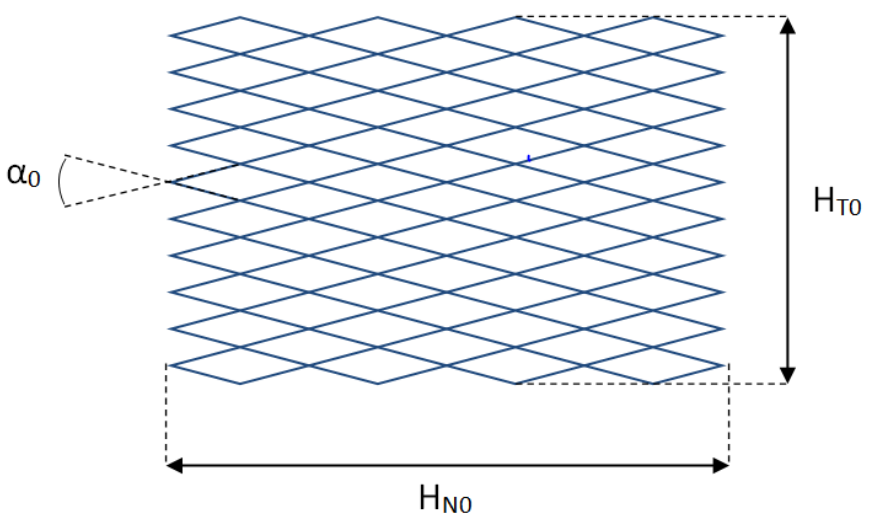

Figure 8: Dimensions $H_{N 0}$ and $H_{T 0}$ of a netting panel allow the characteristic length of the mesh sides and the mesh angle $\alpha_{0}$ at rest to be calculated.

For the single twine netting, the length of mesh sides was $40.44 \pm 0.3$ $\mathrm{mm}$. The samples had 4 and 10 meshes in the N-direction and T-direction respectively (Fig. 8). Ten samples were tested. The mass and the dimensions at rest $H_{N 0}$ and $H_{T 0}$ are given by Table 1 .

For the double twine netting, the length of mesh sides was $49 \pm 0.2 \mathrm{~mm}$. Ten samples, with two different sizes, were tested. The mass, the dimensions at rest $H_{N 0}$ and $H_{T 0}$ for samples with 4 and 10 meshes in the N-direction and T-direction respectively are given by Table 2 , and for samples with 5 and 25 meshes in the N-direction and T-direction respectively are given by Table 3 .

\subsubsection{Breztop polyethylene braided netting}

We tested single twine netting made of Breztop polyethylene. The Breztop polyethylene-fibre braided twine is a product of Le Drezen company. The diameter of the twine was $2.5 \pm 0.1 \mathrm{~mm}$. The length of mesh sides was $40 \pm$ $0.2 \mathrm{~mm}$. The samples had 4 and 10 meshes in the N-direction and T-direction respectively. Ten samples were tested. The mass and the dimensions at rest 


\begin{tabular}{|c|c|c|c|c|c|c|c|}
\hline 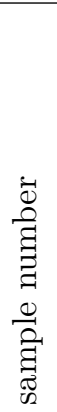 & 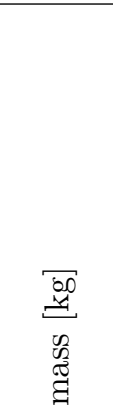 & $\begin{array}{l}\bar{Z} \\
z \\
z\end{array}$ & $\begin{array}{l}\vec{g} \\
\stackrel{E}{*}\end{array}$ & 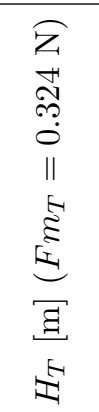 & 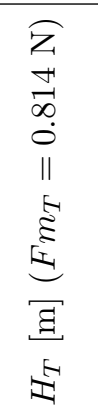 & 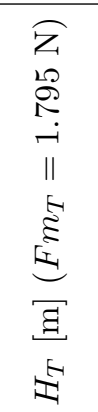 & 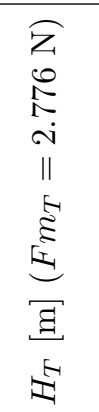 \\
\hline 1 & 0.0614 & 0.320 & 0.097 & 0.201 & 0.294 & 0.436 & 0.505 \\
\hline 2 & 0.0612 & 0.318 & 0.095 & 0.193 & 0.289 & 0.427 & 0.495 \\
\hline 3 & 0.0624 & 0.323 & 0.095 & 0.200 & 0.300 & 0.427 & 0.507 \\
\hline 4 & 0.0622 & 0.320 & 0.100 & 0.197 & 0.289 & 0.430 & 0.501 \\
\hline 5 & 0.0618 & 0.318 & 0.097 & 0.195 & 0.291 & 0.429 & 0.503 \\
\hline 6 & 0.0626 & 0.325 & 0.102 & 0.205 & 0.303 & 0.440 & 0.507 \\
\hline 7 & 0.0618 & 0.323 & 0.103 & 0.212 & 0.304 & 0.433 & 0.498 \\
\hline 8 & 0.0611 & 0.320 & 0.100 & 0.192 & 0.286 & 0.423 & 0.491 \\
\hline 9 & 0.0617 & 0.324 & 0.098 & 0.195 & 0.293 & 0.433 & 0.497 \\
\hline 10 & 0.0614 & 0.320 & 0.098 & 0.197 & 0.288 & 0.435 & 0.513 \\
\hline
\end{tabular}

Table 1: Mass and dimensions of the single twine netting samples of green polyethylene type.

\subsubsection{Brezline polyethylene braided netting}

We investigated single twine netting made of Brezline polyethylene. The Brezline polyethylene-fibre braided twine is a product of Le Drezen company. The diameter of the twine was $4 \pm 0.1 \mathrm{~mm}$. The length of mesh sides was 59.84 $225 \pm 0.6 \mathrm{~mm}$. The samples had 4 and 10 meshes in the $\mathrm{N}$-direction and T-direction respectively. Ten samples were tested. The mass and the dimensions at rest $H_{N 0}$ and $H_{T 0}$ are given by Table 5

\subsubsection{Polyamide braided netting}

We investigated single twine netting made of polyamide braided twines.

${ }_{230}$ The diameter of the twine was $2.32 \pm 0.1 \mathrm{~mm}$. The length of mesh sides was $29.5 \pm 0.6 \mathrm{~mm}$. The samples had 4 and 10 meshes in the N-direction and T- 


\begin{tabular}{|c|c|c|c|c|c|c|c|c|}
\hline 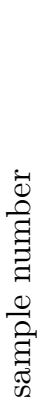 & 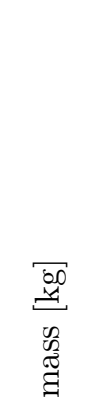 & 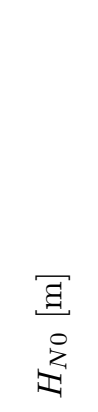 & 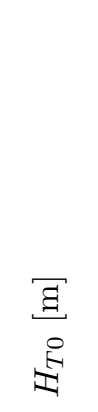 & 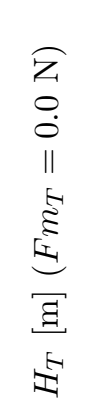 & 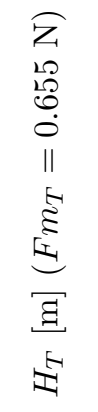 & 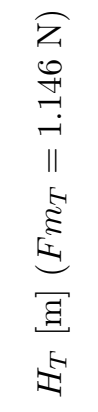 & 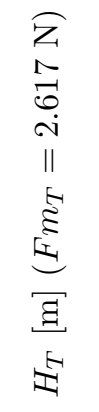 & 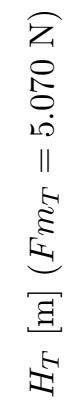 \\
\hline 1 & 0.164 & 0.358 & 0.400 & 0.425 & 0.495 & 0.540 & 0.620 & 0.685 \\
\hline 2 & 0.164 & 0.351 & 0.435 & 0.455 & 0.510 & 0.545 & 0.625 & 0.685 \\
\hline 3 & 0.163 & 0.360 & 0.390 & 0.430 & 0.485 & 0.530 & 0.620 & 0.680 \\
\hline 4 & 0.163 & 0.353 & 0.425 & 0.450 & 0.510 & 0.545 & 0.620 & 0.685 \\
\hline 5 & 0.164 & 0.364 & 0.365 & 0.400 & 0.460 & 0.505 & 0.600 & 0.670 \\
\hline
\end{tabular}

Table 2: Mass and dimensions of the $4 \times 10$-mesh double twine netting samples of green polyethylene type.

\begin{tabular}{|c|c|c|c|c|c|c|c|c|c|}
\hline 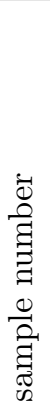 & 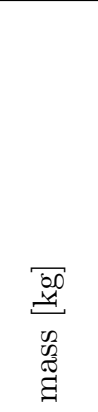 & $\begin{array}{l}\bar{g} \\
\vdots \\
z \\
Z\end{array}$ & $\begin{array}{l}g \\
\mathcal{g} \\
\stackrel{E}{ \pm}\end{array}$ & 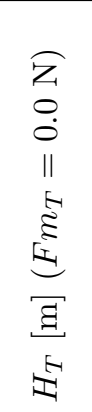 & 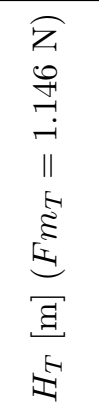 & 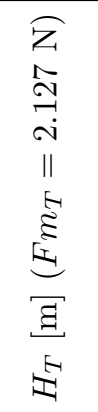 & 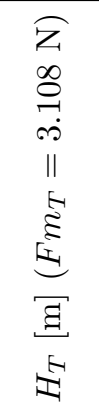 & 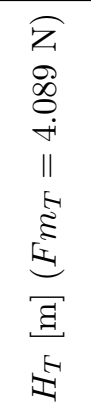 & 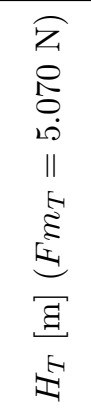 \\
\hline 1 & 0.482 & 0.357 & 0.405 & 0.605 & 0.870 & 1.075 & 1.240 & 1.360 & 1.475 \\
\hline 2 & 0.493 & 0.358 & 0.400 & 0.585 & 0.855 & 1.060 & 1.230 & 1.340 & 1.450 \\
\hline 3 & 0.490 & 0.360 & 0.387 & 0.590 & 0.855 & 1.040 & 1.230 & 1.340 & 1.470 \\
\hline 4 & 0.481 & 0.362 & 0.375 & 0.590 & 0.850 & 1.030 & 1.210 & 1.330 & 1.450 \\
\hline 5 & 0.485 & 0.361 & 0.380 & 0.595 & 0.850 & 1.040 & 1.215 & 1.340 & 1.460 \\
\hline
\end{tabular}

Table 3: Mass and dimensions of the $\mathbf{5 x 2 5}$-mesh double twine netting samples of green polyethylene type. 


\begin{tabular}{|c|c|c|c|c|c|c|c|}
\hline 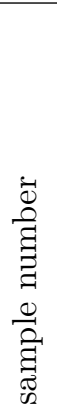 & 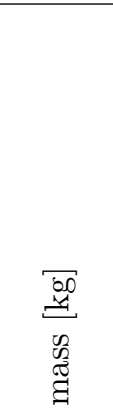 & 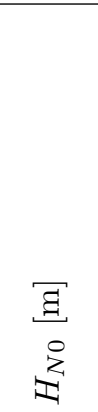 & $\begin{array}{l}\vec{g} \\
\stackrel{E}{*}\end{array}$ & 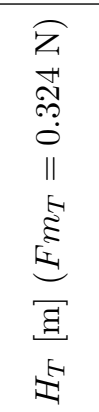 & 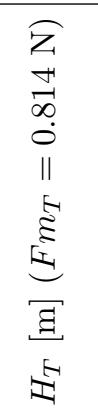 & 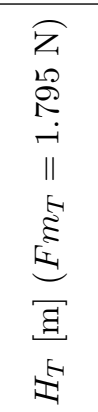 & 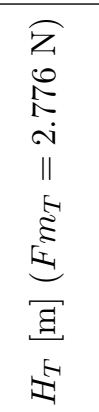 \\
\hline 1 & 0.0184 & 0.318 & 0.080 & 0.407 & 0.558 & 0.624 & 0.648 \\
\hline 2 & 0.0184 & 0.318 & 0.087 & 0.398 & 0.552 & 0.626 & 0.650 \\
\hline 3 & 0.0184 & 0.318 & 0.085 & 0.417 & 0.563 & 0.634 & 0.655 \\
\hline 4 & 0.0183 & 0.317 & 0.087 & 0.423 & 0.568 & 0.629 & 0.653 \\
\hline 5 & 0.0183 & 0.318 & 0.082 & 0.419 & 0.560 & 0.628 & 0.651 \\
\hline 6 & 0.0186 & 0.317 & 0.084 & 0.428 & 0.571 & 0.633 & 0.656 \\
\hline 7 & 0.0184 & 0.317 & 0.090 & 0.414 & 0.563 & 0.629 & 0.651 \\
\hline 8 & 0.0184 & 0.317 & 0.087 & 0.430 & 0.569 & 0.629 & 0.650 \\
\hline 9 & 0.0183 & 0.318 & 0.086 & 0.434 & 0.574 & 0.631 & 0.653 \\
\hline 10 & 0.0184 & 0.318 & 0.085 & 0.424 & 0.567 & 0.626 & 0.650 \\
\hline
\end{tabular}

Table 4: Mass and dimensions of the single twine netting samples of Breztop polyethylene type.

direction respectively. Ten samples were tested. The mass and the dimensions at rest $H_{N 0}$ and $H_{T 0}$ are given by Table 6 .

\subsection{Experiments}

Each rectangular panel of netting was suspended by its top boundary so that the T-direction of the netting was vertical. The panel was subject to its own weight and to forces per mesh $\mathbf{F m}_{\mathbf{T}}$ applied in the T-direction on the knots at the bottom (Fig. 9).

When a netting panel, which had 10 meshes in the T-direction, was suspended, the positions of 5 knots were measured in order to calculate the heights of the four quarters $H 1, H 2, H 3$ and $H 4$ (Fig. 10 left), and the total height of 


\begin{tabular}{|c|c|c|c|c|c|c|c|}
\hline 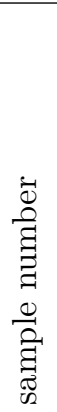 & 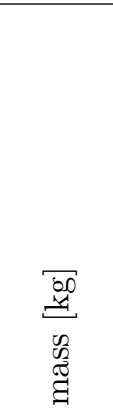 & 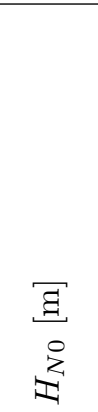 & $\begin{array}{l}\vec{g} \\
\stackrel{E}{*}\end{array}$ & 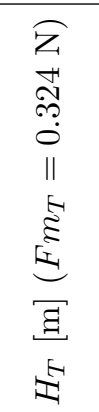 & 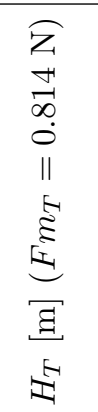 & 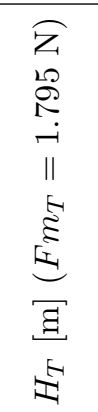 & 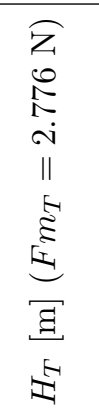 \\
\hline 1 & 0.1165 & 0.470 & 0.138 & 0.370 & 0.509 & 0.702 & 0.802 \\
\hline 2 & 0.1165 & 0.467 & 0.128 & 0.361 & 0.510 & 0.711 & 0.814 \\
\hline 3 & 0.1158 & 0.474 & 0.128 & 0.343 & 0.494 & 0.697 & 0.807 \\
\hline 4 & 0.1189 & 0.479 & 0.125 & 0.343 & 0.501 & 0.707 & 0.816 \\
\hline 5 & 0.1182 & 0.478 & 0.132 & 0.358 & 0.514 & 0.714 & 0.818 \\
\hline 6 & 0.1186 & 0.475 & 0.135 & 0.361 & 0.512 & 0.710 & 0.810 \\
\hline 7 & 0.1154 & 0.472 & 0.126 & 0.346 & 0.495 & 0.691 & 0.797 \\
\hline 8 & 0.1196 & 0.480 & 0.144 & 0.399 & 0.547 & 0.746 & 0.848 \\
\hline 9 & 0.1175 & 0.483 & 0.128 & 0.392 & 0.547 & 0.749 & 0.854 \\
\hline 10 & 0.1187 & 0.480 & 0.133 & 0.379 & 0.532 & 0.737 & 0.849 \\
\hline
\end{tabular}

Table 5: Mass and dimensions of the single twine netting samples of Brezline polyethylene type.

the netting panel $H_{T}$ (Fig. 9p). In the case of the suspension of a netting panel which had 25 meshes in the T-direction, the positions of 6 knots were measured in order to calculate the heights of the five fifths $H 1, H 2, H 3, H 4$ and $H 5$ (Fig. ${ }_{245} 10$ right), and the total height of the netting panel $H_{T}$.

\section{Results}

\subsection{Comparison of numerical and experimental heights}

For each sample, and each loading level, the bending stiffness $E I$ was identified by an inverse identification algorithm minimizing the difference between the numerical and experimental total heights $H_{T}$ of the netting panel. The 


\begin{tabular}{|c|c|c|c|c|c|}
\hline 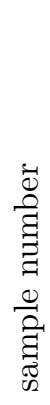 & 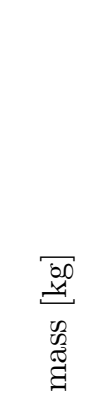 & \begin{tabular}{l}
$\mathbb{Z}$ \\
0 \\
$\mathcal{E}$ \\
\multirow{2}{*}{}
\end{tabular} & 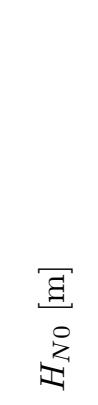 & 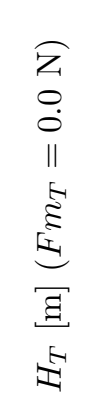 & 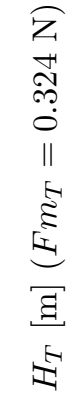 \\
\hline 1 & 0.0209 & 0.222 & 0.125 & 0.264 & 0.427 \\
\hline 2 & 0.0211 & 0.235 & 0.128 & 0.242 & 0.441 \\
\hline 3 & 0.0212 & 0.235 & 0.113 & 0.223 & 0.448 \\
\hline 4 & 0.0208 & 0.227 & 0.115 & 0.205 & 0.429 \\
\hline 5 & 0.0212 & 0.235 & 0.112 & 0.196 & 0.446 \\
\hline 6 & 0.0211 & 0.232 & 0.110 & 0.198 & 0.436 \\
\hline 7 & 0.0607 & 0.225 & 0.105 & 0.250 & 0.428 \\
\hline 8 & 0.0612 & 0.235 & 0.130 & 0.265 & 0.442 \\
\hline 9 & 0.0210 & 0.232 & 0.113 & 0.242 & 0.435 \\
\hline 10 & 0.0209 & 0.235 & 0.125 & 0.235 & 0.447 \\
\hline
\end{tabular}

Table 6: Mass and dimensions of the single twine netting samples of polyamide type.

algorithm was based on the dichotomy method. The measurements of the total heights $H_{T}$ of the netting panels, subject to different levels of force per mesh $F m_{T}$, are given in Tables 16 .

Once the bending stiffness was identified, we compared the experimental and numerical heights $H 1, H 2, H 3$ and $H 4$ for a 4 x10-mesh netting sample and $H 1$, $H 2, H 3, H 4$ and $H 5$ for a $5 \times 25$-mesh netting sample. The bending stiffness was assumed to be the same in the whole netting sample.

Figures 11 to 16 show the numerical and experimental heights for each sample and each loading level. 


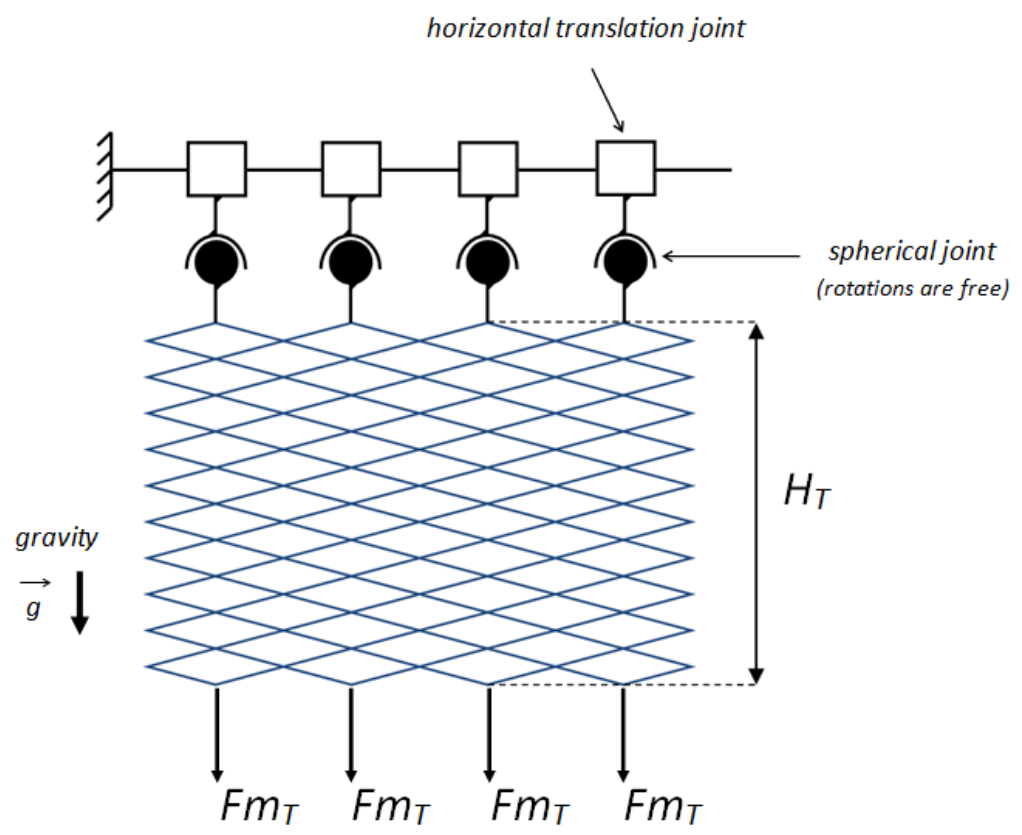

Figure 9: Experimental setup plan. The panel is suspended from one of its ends and is subject to its own weight and to forces $F m_{T}$ applied on bottom knots. $F m_{T}$ is called the force per mesh.

\subsection{Bending stiffness}

Figures 17 to 22 present the evolution of the bending stiffness $E I$, identified as explained in Section 4.1, as a function of the applied load $F m_{T}$. These figures also present the coefficient of variation (ratio of the standard deviation to the mean) of the bending stiffness $E I$ as a function of the applied load $F m_{T}$.

Fig. 23 shows the evolution of the bending stiffness $E I$ as a function of the applied load $F m_{T}$ in the cases of 4x10-mesh and 5x25-mesh double twine green PE netting samples. 

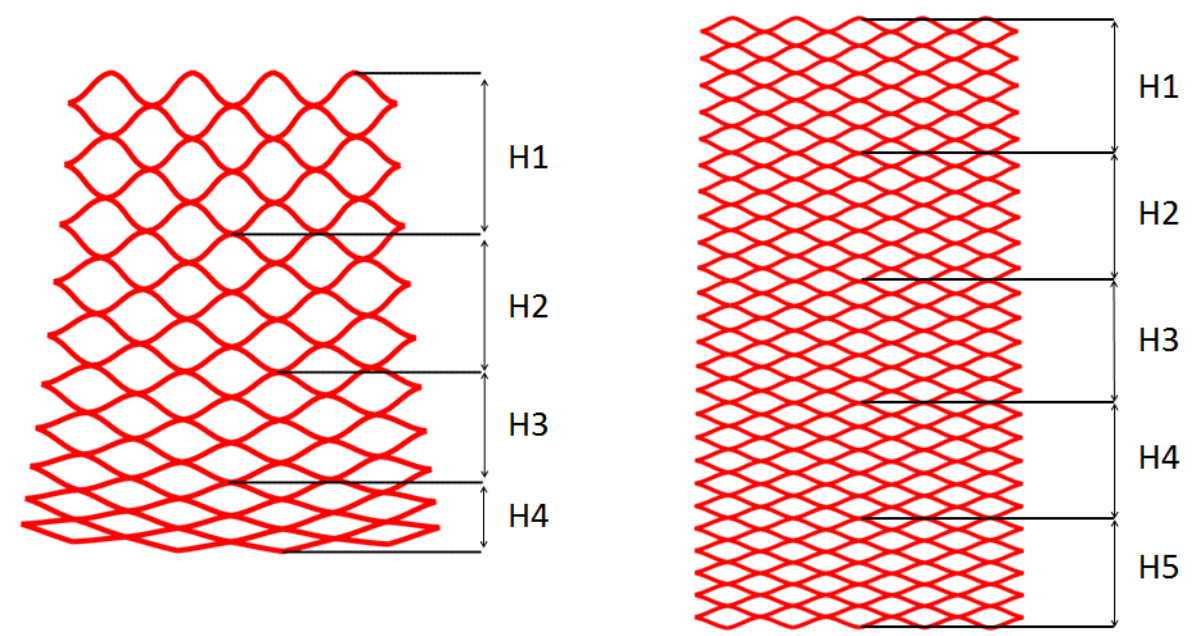

Figure 10: Definition of heights for a 4x10-mesh netting panel (left) and a 5x25-mesh netting panel (right). The heights are measured vertically. The total height $H_{T}$ of the panel is the sum of $\mathrm{H} 1, \mathrm{H} 2, \mathrm{H} 3$ and $\mathrm{H} 4$ for a 4x10-mesh netting panel, and the sum of $\mathrm{H} 1, \mathrm{H} 2, \mathrm{H} 3, \mathrm{H} 4$ and $\mathrm{H} 5$ for a $5 \times 25$-mesh netting panel.

\section{Discussion}

In Figures 11 to 16 , it can be seen that the heights $(H 1$ to $H 5)$ increase when the applied load $F m_{T}$ increases, as expected. It can be also seen that for each loading level $F m_{T}$, the height at the top quarter $H 1$ (or fifth) is higher than at the bottom quarter $H 4$ (or $H 5$ ). This point shows that the deformation of the netting is not uniform, which is due to gravity.

We also see that the numerical model gives height values similar to experiments for all tested types of fishing net, even if the height decreases noticeably from the top quarter $H 1$ (or fifth) to the bottom quarter $H 4$ (or H5) (Fig. 13 , 15 and 16). Thus, the finite element model presented in this paper captures the heterogeneous deformation field of the netting samples during these suspending tests.

Figures 17 to 22 show the evolution of the bending stiffness $E I$, identified using the finite element model, as a function of the applied force per mesh $F m_{T}$. When the force $F m_{T}$ and thus the mesh opening increase, the tensile forces in 


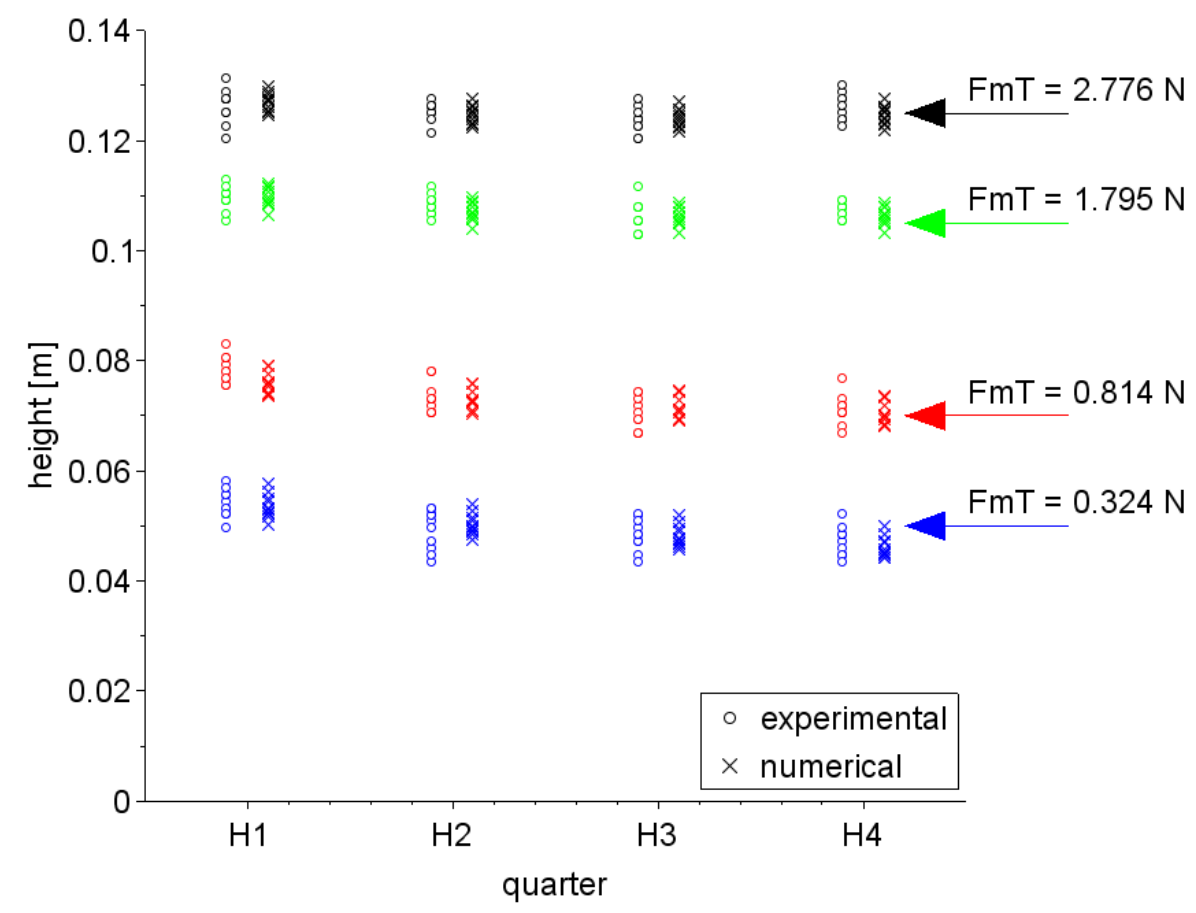

Figure 11: Single twine green PE netting, 4x10-mesh sample. Results of experimental suspending tests and their numerical simulations; height of the quarters of ten netting samples for 4 loading levels $F m_{T}$. Abscissa H1 to H4 are defined in Fig. 10

the mesh sides increase so we may expect the twine diameter to decrease. A smaller diameter means a smaller moment of inertia $I$. Thus, we may expect a decrease in $E I$ when the loading level increases. However, the results do not show any decrease in $E I$ when $F m_{T}$ increases. In the cases of double twine green PE netting (Fig. 18), single twine Breztop PE netting (Fig. 20) and single twine PA netting (Fig. 22), we note an increase in the bending stiffness. It could be due to the complexity of the twine braided structure: when the loading level increases, the shear force and bending moment through the twine section increase, so the shape of the section may change drastically and this could affect the bending stiffness $E I$.

Moreover, Fig. 23 shows that in the case of double twine green PE netting, 


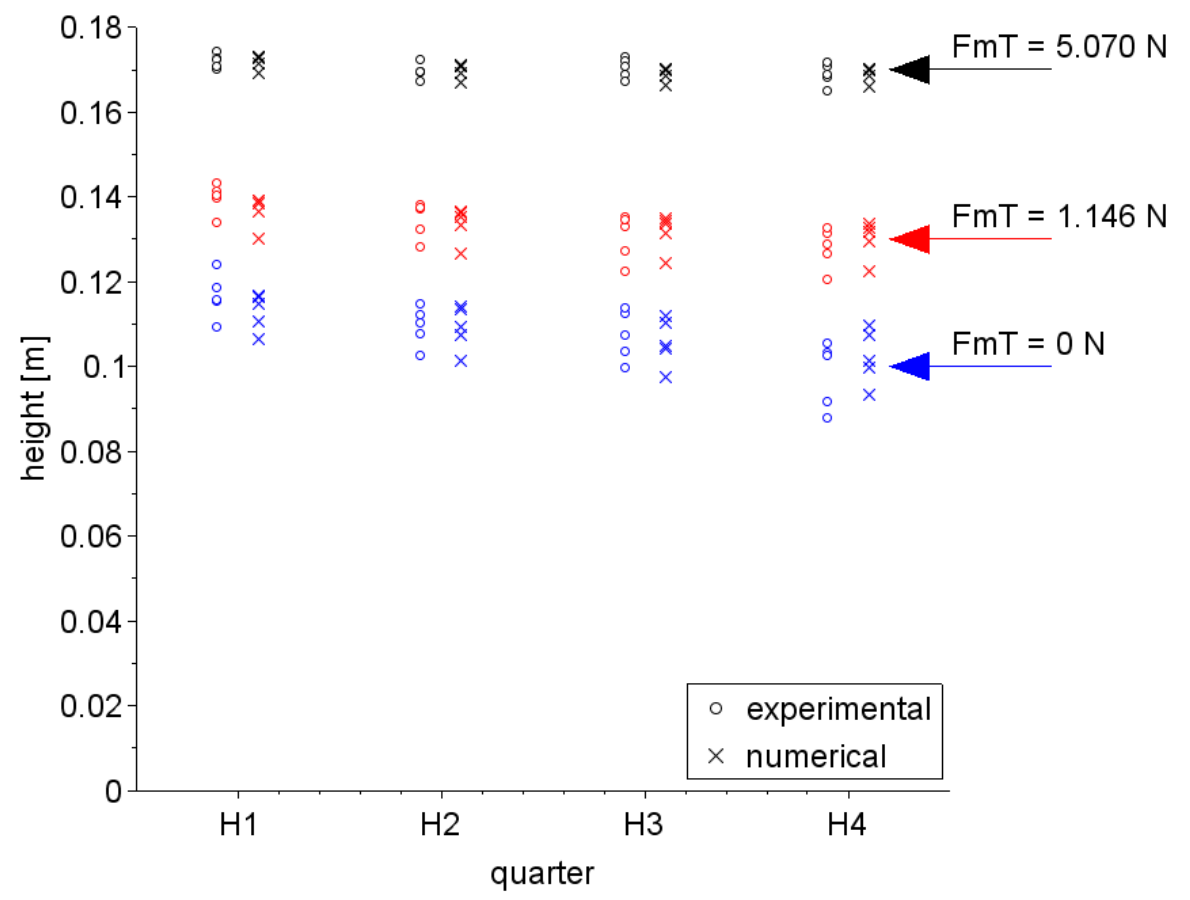

Figure 12: Double twine green PE netting, 4x10-mesh sample. Results of experimental suspending tests and their numerical simulations; height of the quarters of five netting samples for 3 loading levels $F m_{T}$.

the bending stiffness $E I$ does not depend on the size of the sample. Indeed, for $4 \times 10$ - and $5 \times 25$-mesh netting samples, the evaluated bending stiffnesses are similar, whatever the loading level.

Figures 17 to 22 also show that the coefficient of variation in $E I$ is the highest when the value of $F m_{T}$ is close to zero. In case of higher loading level, the coefficient of variation is below $10 \%$.

Here, the loading is applied only in the T-direction. The method does not investigate the effect of significant loading in the N-direction. It could be worth investigating coupled N- and T-direction loading effects. For such an investigation, the present experimental method is not suitable, or it has to be used in conjunction with another experimental protocol such as that used by Sala et al. 


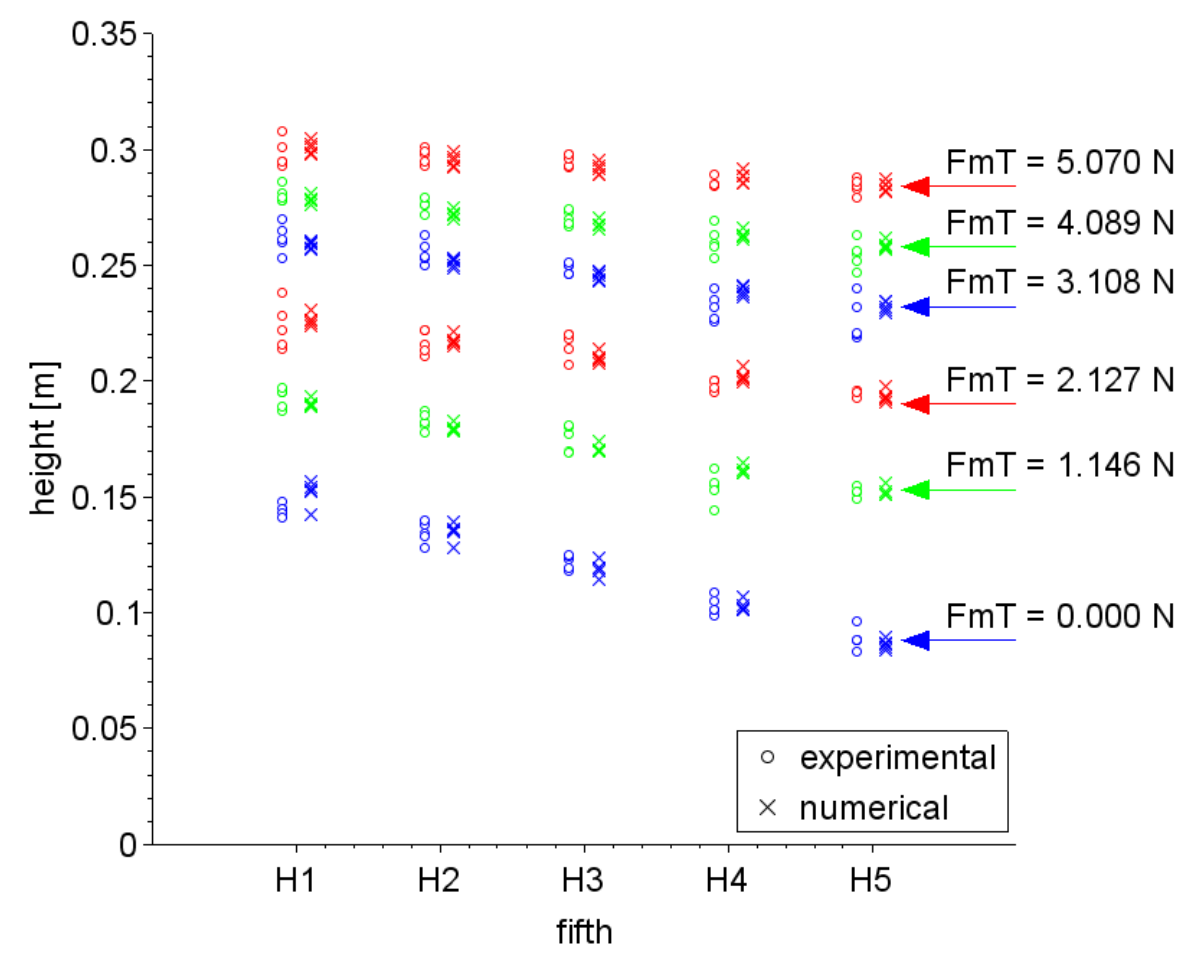

Figure 13: Double twine green PE netting, 5x25-mesh sample. Results of experimental suspending tests and their numerical simulations; height of the fifths of five netting samples for 6 loading levels $F m_{T}$.

\section{Conclusion}

In this paper, we propose a numerical model which is applicable to nonuniform deformation or non-closed meshes. The bending stiffness in the numerical model was evaluated by an inverse identification algorithm so that the total

height of the suspended netting panel given by the numerical model equalled to the measured height. The evolution of the mesh stretch along the suspended samples, from the top to the bottom, seems to be captured by the model. The proposed procedure resulted in the evaluation of the mesh resistance to opening 


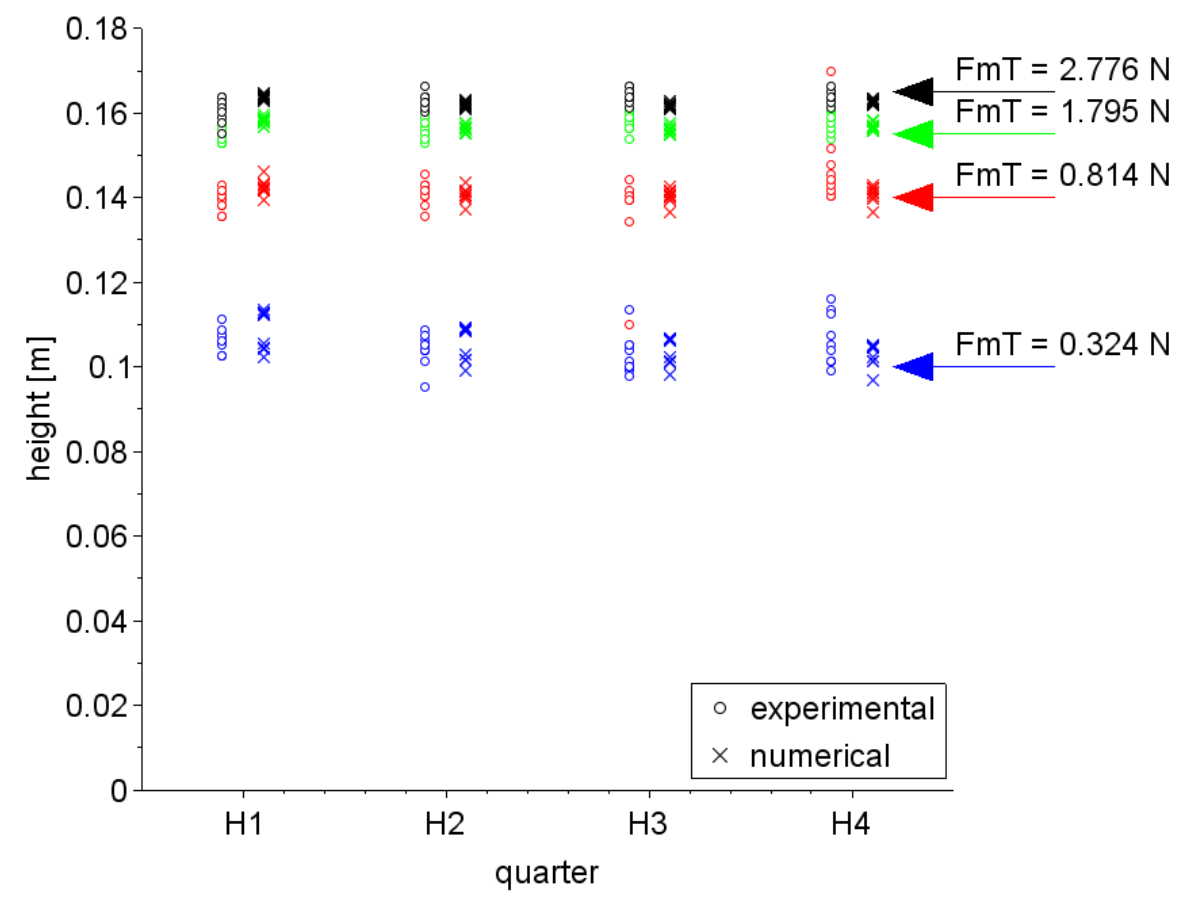

Figure 14: Single twine Breztop PE netting, 4x10-mesh sample. Results of experimental suspending tests and their numerical simulations; height of the quarters of ten netting samples for 4 loading levels $F m_{T}$.

of the tested fishing nets as a function of the level of loading.

The ratio of standard deviation to mean of the evaluated bending stiffness, for all types of tested netting, is below 10\%, except when the netting sample was solely under its own weight. Furthermore, in some instances the bending stiffness is independent of the sample size. In some cases, it is clearly seen that the bending stiffness increases when the applied loading increases.

The proposed finite element model aims to be more versatile than the existing models of mesh resistance to opening: it allows the simulation of fishing nets with non-uniform and large deformation. This software tool is open-source.

In the future, the authors will take into account the size of the knots and will study the influence of this on the identified bending stiffness. The method 


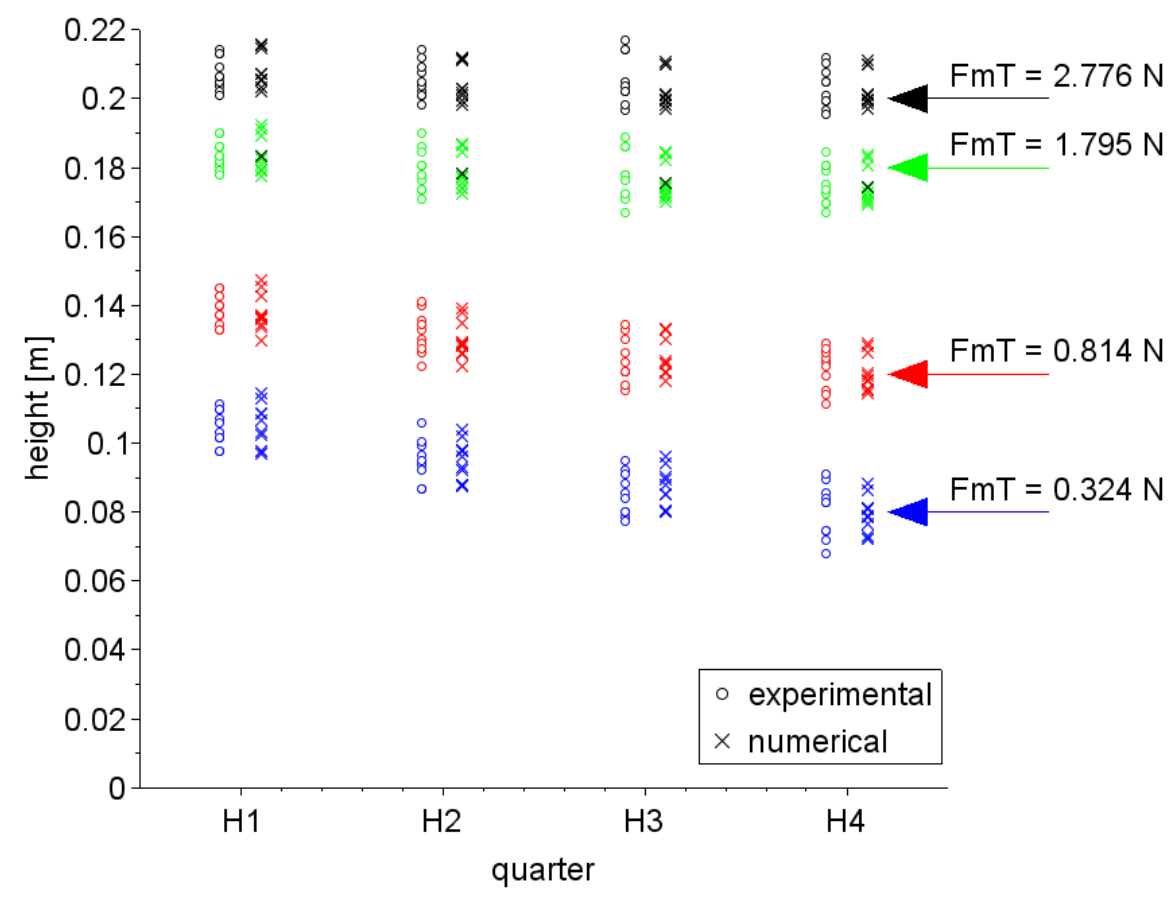

Figure 15: Single twine Brezline PE netting, 4x10-mesh sample. Results of experimental suspending tests and their numerical simulations; height of the quarters of ten netting samples for 4 loading levels $F m_{T}$.

involving suspending tests and the use of the proposed finite element model is expected to be compared with other existing methods. Further work should study the effect of the viscosity of the material by testing and measuring, for example, netting samples loaded with creep and relaxation stages. 


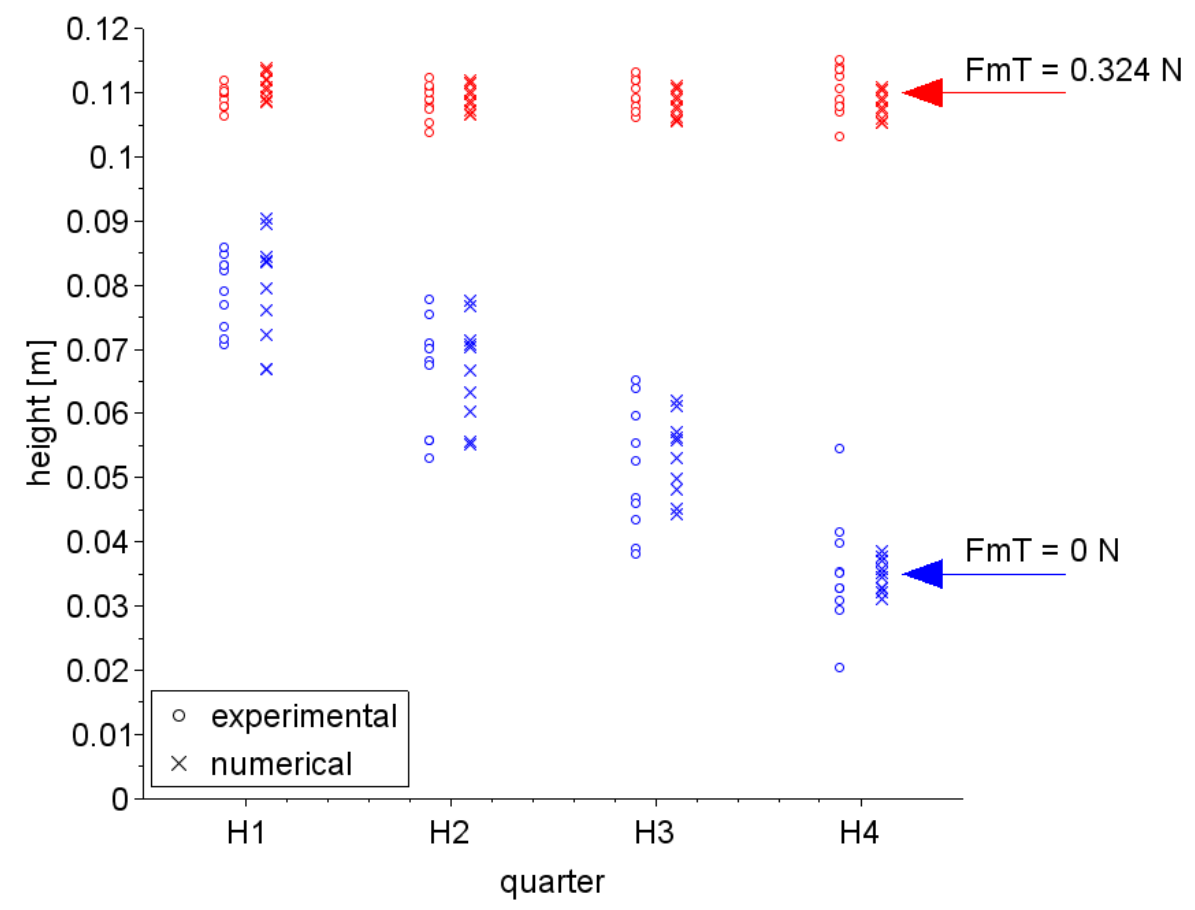

Figure 16: Single twine PA netting, 4x10-mesh sample. Results of experimental suspending tests and their numerical simulations; height of the quarters of ten netting samples for 2 loading levels $F m_{T}$. 


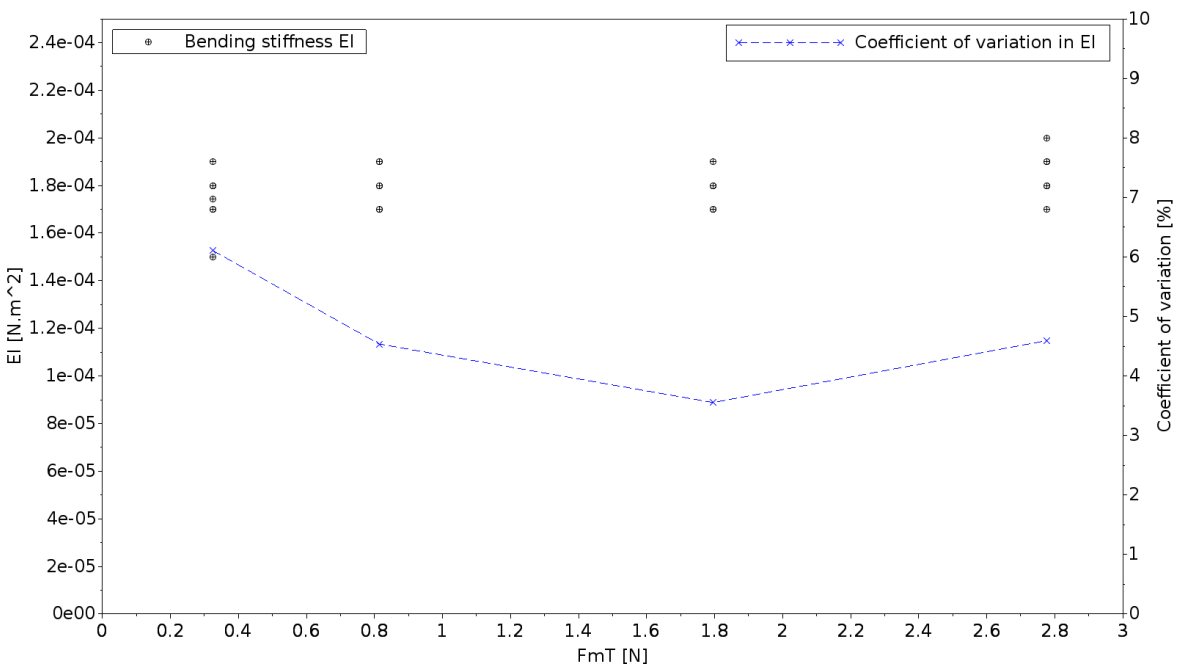

Figure 17: Single twine green PE netting, 4x10-mesh sample. Results of the numerical model identified on the experimental suspending tests; evolutions of the bending stiffness $E I$ and its coefficient of variation (ratio of the standard deviation to the mean) as a function of the applied load $F m_{T}$, for ten netting samples. 


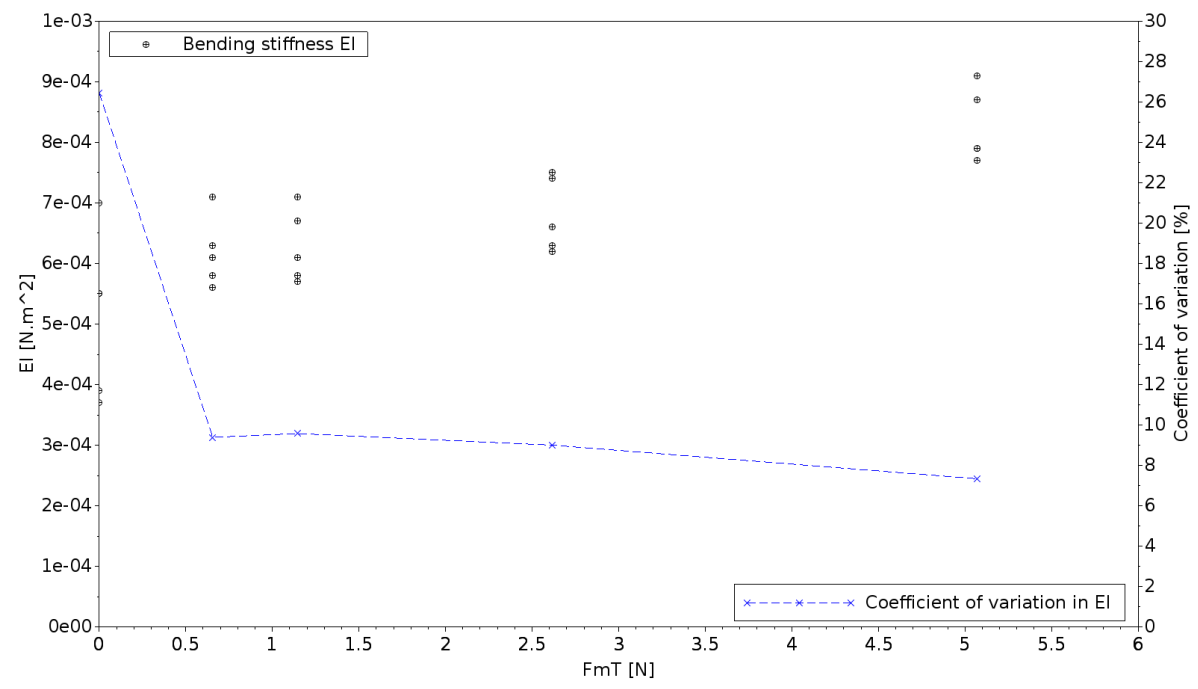

Figure 18: Double twine green PE netting, 4x10-mesh sample. Results of the numerical model identified on the experimental suspending tests; evolutions of the bending stiffness $E I$ and its coefficient of variation (ratio of the standard deviation to the mean) as a function of the applied load $F m_{T}$, for five netting samples. 


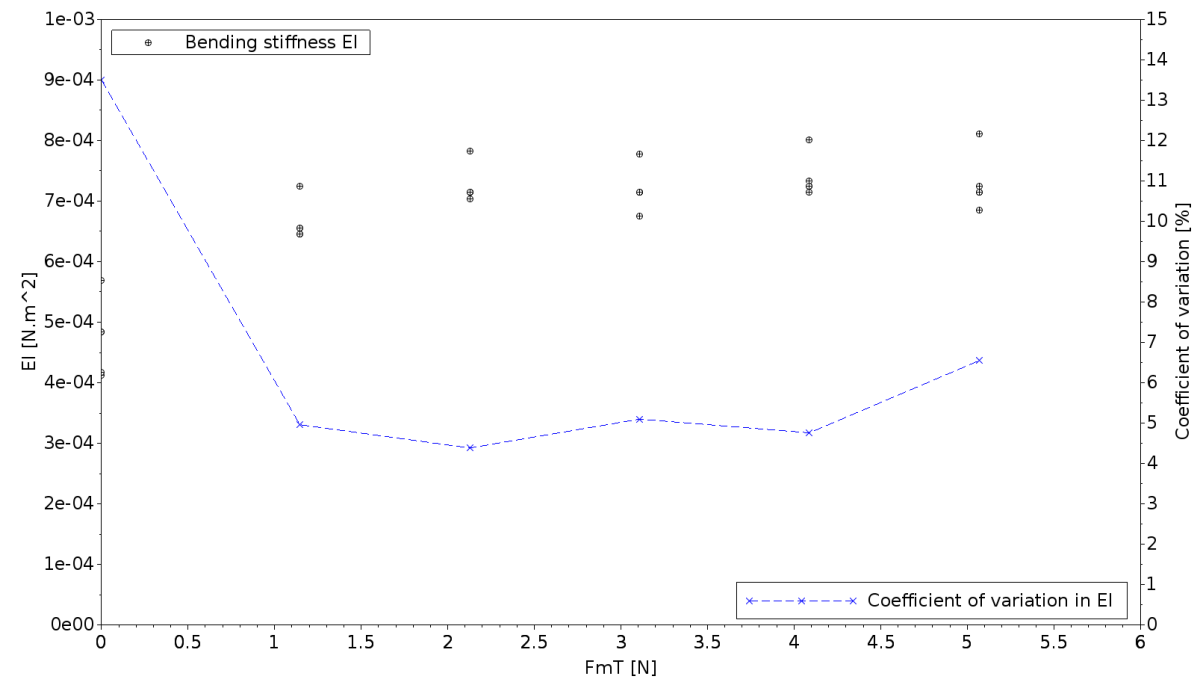

Figure 19: Double twine green PE netting, 5x25-mesh sample. Results of the numerical model identified on the experimental suspending tests; evolutions of the bending stiffness $E I$ and its coefficient of variation (ratio of the standard deviation to the mean) as a function of the applied load $F m_{T}$, for five netting samples. 


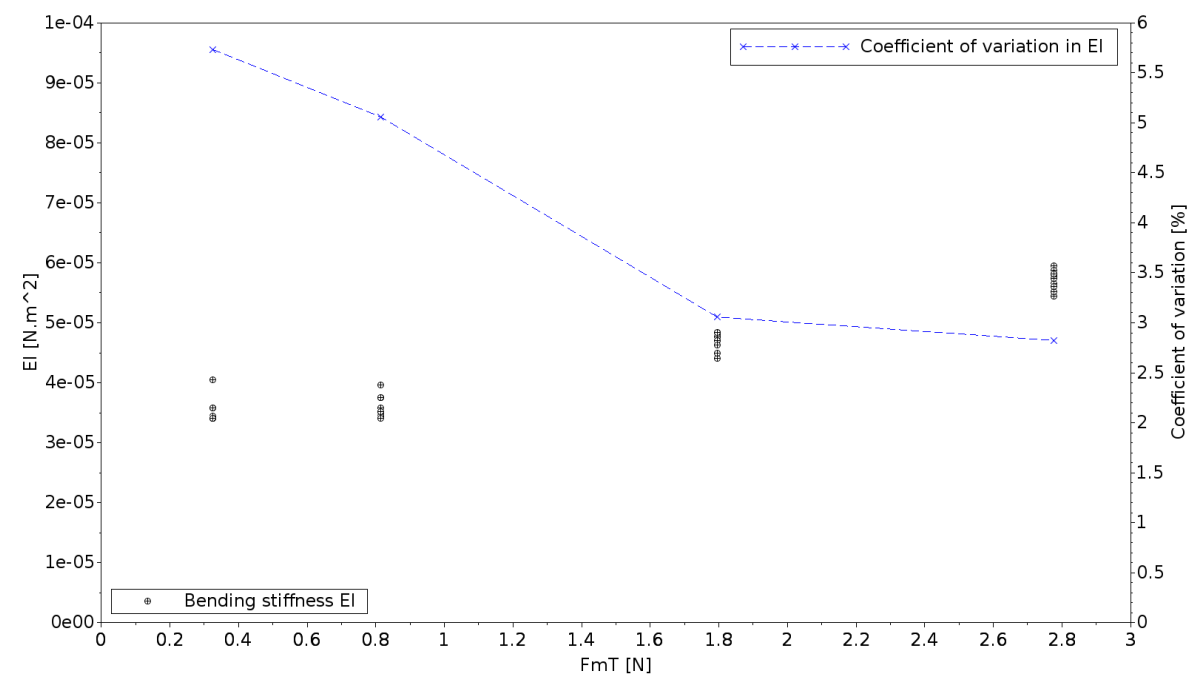

Figure 20: Single twine Breztop PE netting, 4x10-mesh sample. Results of the numerical model identified on the experimental suspending tests; evolutions of the bending stiffness $E I$ and its coefficient of variation (ratio of the standard deviation to the mean) as a function of the applied load $F m_{T}$, for ten netting samples. 


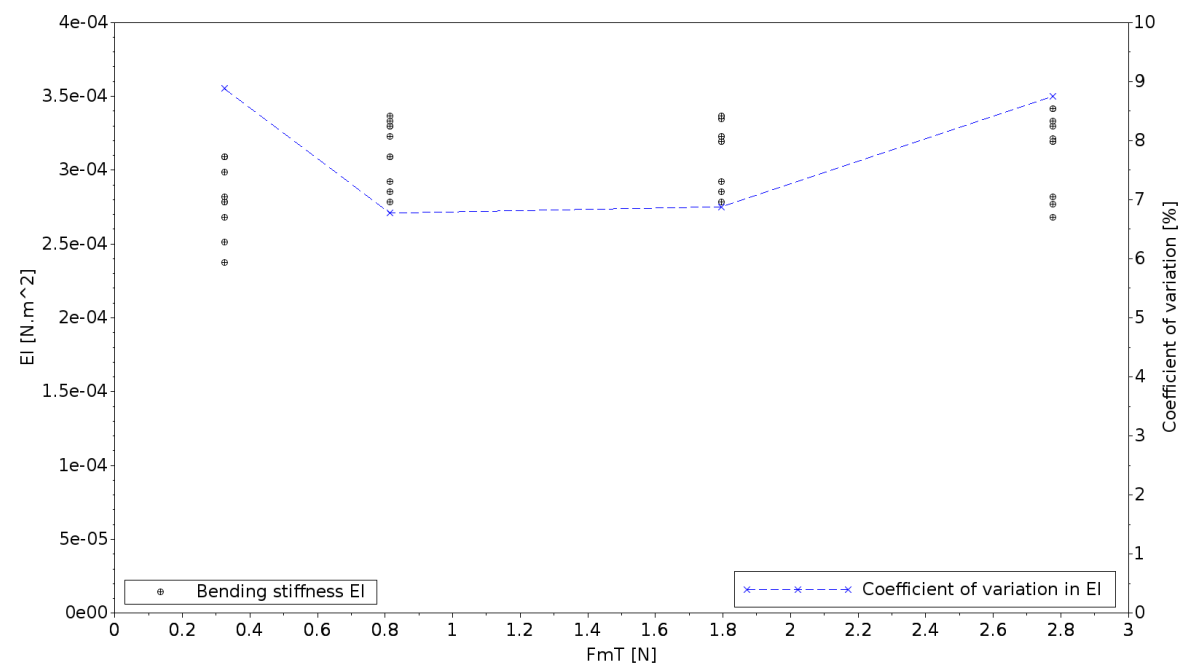

Figure 21: Single twine Brezline PE netting, 4x10-mesh sample. Results of the numerical model identified on the experimental suspending tests; evolutions of the bending stiffness $E I$ and its coefficient of variation (ratio of the standard deviation to the mean) as a function of the applied load $F m_{T}$, for ten netting samples. 


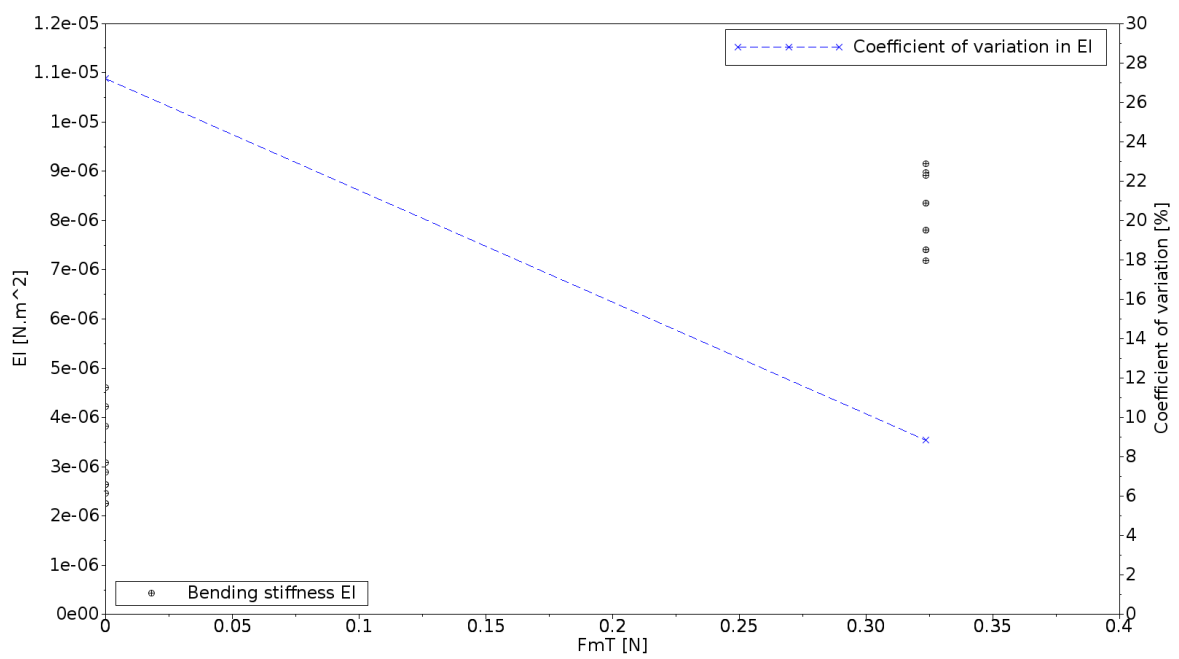

Figure 22: Single twine PA netting, 4x10-mesh sample. Results of the numerical model identified on the experimental suspending tests; evolutions of the bending stiffness $E I$ and its coefficient of variation (ratio of the standard deviation to the mean) as a function of the applied load $\mathrm{Fm}_{T}$, for ten netting samples. 


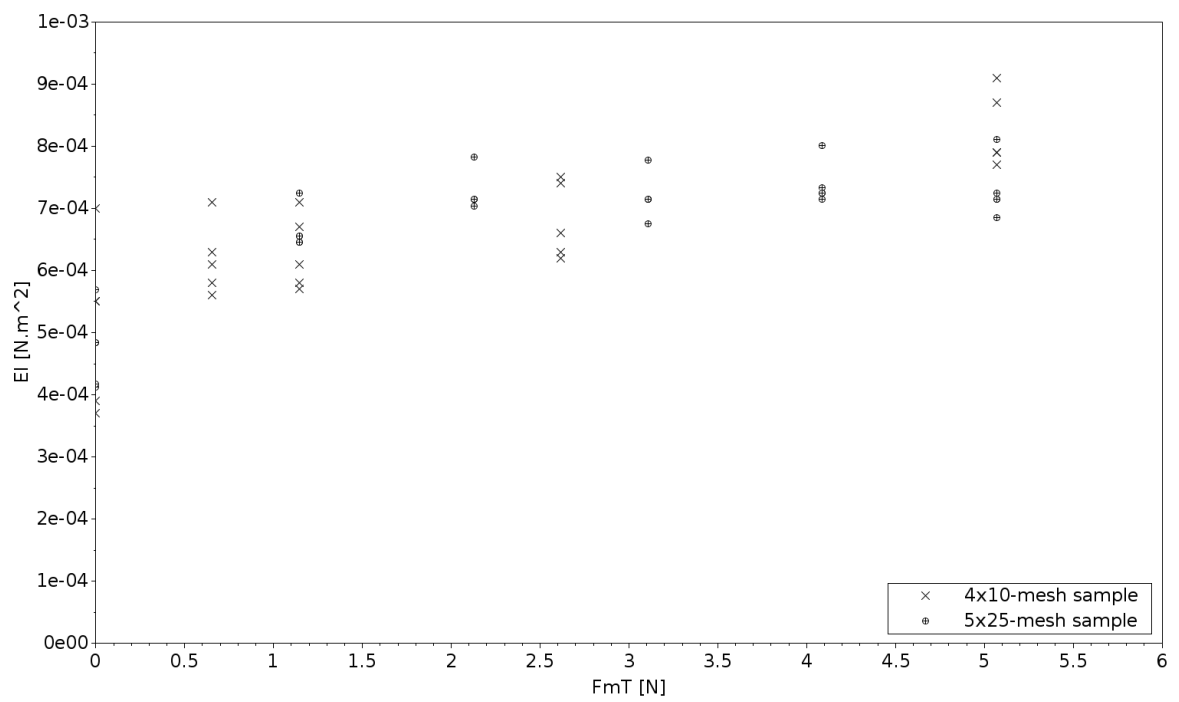

Figure 23: Double twine green PE netting. Results of the numerical model identified on the experimental suspending tests; evolutions of the bending stiffness $E I$ as a function of the applied load $F m_{T}$, for five 4x10-mesh netting samples and five 5x25-mesh netting samples. This shows that the bending stiffness is independent of the size of the panels $(4 \times 10-$ and $5 \times 25$-mesh netting samples). 


\section{Acknowledgements}

(Région Bretagne) under a financing agreement for doctoral research (no. 13/2.213

\section{References}

Balash, C., Sterling, D., Binns, J., Thomas, G., Bose, N., 2016. Drag characterisation of prawn-trawl bodies. Ocean Engineering 113, 18-23.

Boerema, L.K., 1956. Some experiments on factors influencing mesh selection in trawls. ICES Journal of Marine Science 21 (2), 175-191.

Broadhurst, M.K., Sterling, D.J., Millar, R.B., 2015. Increasing lateral mesh openings in penaeid trawls to improve selection and reduce drag. Fisheries Research 170, 68-75.

Herrmann, B., O’Neill, F.G., 2006. Theoretical study of the influence of twine thickness on haddock selectivity in diamond mesh cod-ends. Fisheries research $80,221-229$.

Herrmann, B., Wienbeck, H., Moderhak, W., Stepputtis, D., Krag, L.A., 2013. The influence of twine thickness, twine number and netting orientation on codend selectivity. Fisheries research 145, 22-36.

Herrmann, B., Wienbeck, H., Stepputtis, D., Ahm Krag, L., Feekings, J., Moderhak, W., 2015. Size selection in codends made of thin-twined dyneema netting compared to standard codends: A case study with cod, plaice and flounder. Fisheries research 167, 82-91.

ISO, 1974. Fishing nets — netting — basic terms and definitions, in: International Standard Organisation 1107, ISO Geneva.

Johnson, A., Balash, C., 2015. Experimental modelling of transverse oscillations in aquaculture netting parallel to the flow - sounds baffling. Journal of China Ocean Engineering 29, 391-400. 
Moderhak, W., 2007. Influence of twine parameters on the shapes of meshes and t90 codends, in: International Workshop Methods for the Development and Evaluation of Maritime Technologies (DEMaT'07), ROSTOCK, Germany.

O'Neill, F., 2002. Bending of twines and fibres under tension. Journal of the Textile Institute 93, 1-10.

O'Neill, F., 2003. A theoretical study of the factors which influence the measurement of fishing netting mesh size. Ocean engineering 30, 2053-2063.

Ortega, J.M., Rheinboldt, W.C., 2000. Iterative Solution Of Nonlinear Equations in Several Variables. Society for Industrial and Applied Mathematics. doi $10.1137 / 1.9780898719468$

De la Prada, A., Gonzales, M., 2015. Quantifying mesh resistance to opening of netting panels: experimental method, regression models, and parameter 口 estimation strategies. ICES Journal of Marine Science 72(2), 697-707. doi:10. 1093/icesjms/fsu125

Priour, D., 2013. A finite element method for netting: application to fish cages and fishing gear. 1st ed., Springer.

Priour, D., Cognard, J.Y., 2011. Investigation of methods for the assessment of the flexural stiffness of netting panels, in: 10th International Workshop Methods for the Development and Evaluation of Maritime Technologies (DEMaT'11), SPLIT, Croatie.

Robertson, J., Stewart, P., 1988. A comparison of size selection of haddock and whiting by square and diamond mesh codends. J. Cons. Int. Explor. Mer 44, $148-161$.

Sala, A., O’Neill, F., Buglioni, G., Lucchetti, A., Palumbo, V., Fryer, R., 2007. Experimental method for quantifying resistance to the opening of netting panels. ICES Journal of Marine Science: Journal du Conseil 64, 1573-1578. 